\title{
It's Time to Add Electrocardiography and Echocardiography to CVD Risk Prediction Models: Results from a Prospective Cohort Study
}

\section{Zhao Li $\mathbb{B D}^{\prime}$ \\ Yiqing Yang' \\ Liqiang Zheng ${ }^{2}$ \\ Guozhe Sun' \\ Xiaofan Guo' \\ Yingxian Sun'}

'Department of Cardiology, The First Hospital of China Medical University, Shenyang, I I000 I, People's Republic of China; ${ }^{2}$ Department of Clinical Epidemiology, Library, Department of Health Policy and Hospital Management, Shengjing Hospital of China Medical University, Shenyang, I 10004, People's Republic of China
Correspondence: Yiqing Yang; Yingxian

Sun

Tel +862483282688

Fax +86 2483282346

Email yangyiqing0725@163.com;

yingxiansun123@163.com
Objective: To develop and validate a new prediction model for the general population based on a large panel of both traditional and novel factors in cardiovascular disease (CVD).

Design and Setting: We used a prospective cohort in the Northeast China Rural Cardiovascular Health Study (NCRCHS).

Participants: A total of 11,956 participants aged $\geq 35$ years were recruited between 2012 and 2013, using a multistage, randomly stratified, cluster-sampling scheme. In 2015 and 2017, the participants were invited to join the follow-up study for incident cardiovascular events. The loss to follow-up number was 351. At the study's end, we obtained the CVD outcome events for 10,349 participants.

Primary and Secondary Outcome Measures: The prediction model was developed using demographic factors, blood biochemical indicators, electrocardiographic (ECG) characteristics, and echocardiography indicators collected at baseline (Model 1). Framinghamrelated variables, namely age, sex, smoking, total and high-density lipoprotein cholesterol and diabetes status were used to construct the traditional model (Model 2).

Results: For the observed population $(n=10,349)$, the median follow-up time was 4.66 years. The total incidence of CVD was $1.1 \%$ year, including stroke $(n=342)$ and coronary heart disease $(n=175)$. The results of Model 1 indicated that in addition to the traditional risk factors, QT interval ( $\mathrm{p}<0.001)$, aortic root diameter $(\mathrm{p}<0.001)$, and ventricular septal thickness $(\mathrm{p}<0.001)$ were predictive factors for CVD. Decision curve analysis (DCA) showed that the net benefit with Model 1 was higher than that of Model 2.

Conclusion: QT interval from electrocardiography and aortic root diameter and ventricular septal thickness from echocardiography should be included in the CVD risk prediction models. Keywords: CVD, predictive model, general cohort, QT interval, aortic root diameter, ventricular septal thickness

\section{Introduction}

Between 1956 and 1966, investigators in Framingham, Massachusetts identified age, hypertension, smoking, and hyperlipidemia as major determinants of coronary heart disease, and coined the term "coronary risk factors". ${ }^{1-3}$ Over time, these traditional markers were codified into risk scores for assessing cardiovascular disease (CVD). ${ }^{4-6}$ Framingham risk score (FRS) mainly applies to white Americans between the 30 and 62 years old and has become one of the most widely used risk assessment tools. However, BMI and family history are not considered in the score, which may underestimate the morbidity and mortality of 
high-risk groups. In addition, some study suggests that FRS models overestimate the risk of coronary heart disease (CHD) in the European population. ${ }^{7}$ Systematic Coronary Risk Evaluation (SCORE) ${ }^{8}$ mainly applies to Europeans aged 40-65 years old. Geographical distribution is added to make the assessment more rigorous, but the clinical outcome index is fatal CVD events, so there may be some limitations for predicts non-fatal CVD. The China-PAR model ${ }^{9}$ is designed for Chinese risk assessment of atherosclerotic cardiovascular disease (ASCVD). It not only includes the sex, age, untreated systolic blood pressure, total cholesterol, high-density lipoprotein cholesterol, waist circumference, smoking, diabetes and other risk factors, but also includes north and south region, urban and rural areas, ASCVD family history. However, although the importance of conventional factors is wellestablished, studies have concluded that more than $50 \%$ of patients with CVD lack the traditional risk factors. ${ }^{10,11}$ This suggests that additional factors play a significant role in the development of CVD.

The increasing availability of cardiac examinations over the past half-century, including the convenience of laboratory evaluations and popularization of electrocardiography (ECG) and echocardiography, has offered possibilities for researchers to detect novel CVD risk factors. For example, a Chinese study has identified that Serum gamma-glutamyl transferase (GGT) are closely related to CVD. ${ }^{12}$ Dekker reported that long QT interval on an ECG is an independent risk factor for coronary heart disease and CVD mortality in healthy people, and other researchers have confirmed that heart rate is associated with CVD. ${ }^{13-15}$ Similarly, left ventricular hypertrophy seen on echocardiography is an independent predictor of incident CVD. ${ }^{16,17}$ Nonetheless, cardiovascular prediction algorithms still largely rely on traditional risk factors; there is currently no new predictive model that integrates the highest possible number of indicators obtained in routine clinical examinations like electrocardiography and echocardiography.

The Northeast China Rural Cardiovascular Health Study (NCRCHS) is a community-based prospective cohort study designed to investigate the risk factors and intervention methods for CVD in the general population. Here, we report the updated incidence of CVD in the NCRCHS cohort, and explore a new cardiovascular predictive model that integrates relevant demographic factors, blood biochemical indicators, ECG characteristics, and echocardiography indicators.

\section{Methods}

\section{Study Population}

The NCRCHS involved rural areas of Northeast China, and its design and inclusion criteria were described previously. ${ }^{18}$ Briefly, 11,956 participants aged $\geq 35$ years were recruited from Dawa, Zhangwu, and Liaoyang counties in Liaoning province between 2012 and 2013, using a multi-stage, randomly-stratified, cluster-sampling scheme. Participants who were pregnant, or who had malignant tumors, malnutrition, hepatic disease, and/or mental disorders were excluded from the present study. Detailed information was collected at baseline for each participant, namely demographic characteristics, blood biochemical indicators, ECG characteristics, and echocardiography indicators. In 2015 and 2017, we invited participants to be involved in a follow-up study, to which 10,700 of the original 11,956 subjects consented and qualified. A total of 10,349 participants (86.6\%) completed at least one follow-up visit. This study was approved by the Ethics Committee of China Medical University (Shenyang, China); ID: 2018194. Written informed consent was obtained from all participants; illiterate participants had their consents signed by close relatives. This study was conducted in accordance with the Declaration of Helsinki.

The cohort was divided into a primary cohort and a validation cohort according to region, with the primary set constituting patients from Dawa and Liaoyang, and the validation set constituting patients from Zhangwu.

\section{Study Variables and Data Collection}

At baseline, detailed information on the patients' demographic characteristics, dietary and lifestyle factors, and medical history was obtained through interviews using a standardized validated questionnaire.

Smoking and drinking statuses were defined as current use. A history of stroke, coronary heart disease, or heart failure at baseline was self-reported and confirmed by medical records. Weight and height were measured with participants wearing lightweight clothing and no shoes. Waist circumference (WC) was measured at the umbilicus using a nonelastic tape. Body mass index (BMI) was computed as weight in kilograms divided by the square of the height in meters. Blood pressure was assessed three times (mean of all three measurements) with participants seated after at least 5 minutes of rest using a standardized automatic electronic sphygmomanometer (HEM-907; 
Omron, Tokyo, Japan). Hypertension was defined as systolic blood pressure (SBP) $\geq 140 \mathrm{~mm} \mathrm{Hg}$, diastolic blood pressure (DBP) $\geq 90 \mathrm{~mm} \mathrm{Hg}$, or the use of antihypertensive medications. ${ }^{19}$ Blood samples were collected in the morning from all participants to measure white blood cell count, total red blood cells, mean red blood cell volume, hemoglobin, plateletcrit (PCT), hematocrit (HCT), and mean corpuscular hemoglobin $(\mathrm{MCH})$ after $12 \mathrm{~h}$ of fasting.

Alanine aminotransferase (ALT) and aspartate aminotransferase (AST), fasting plasma glucose (FPG), total cholesterol (TC), low-density lipoprotein cholesterol (LDL-C), high-density lipoprotein cholesterol (HDL-C), triglyceride (TG), serum creatinine $(\mathrm{SCr})$, blood urea nitrogen (BUN), uric acid (UA), serum chlorine (SCl), serum calcium (SCa), serum phosphorus (SP), serum magnesium $(\mathrm{SMg})$, and serum sodium $(\mathrm{SNa})$ were analyzed enzymatically. Estimated glomerular filtration rate (eGFR) was calculated using the Chronic Kidney Disease Epidemiology Collaboration equation. ${ }^{20}$

All laboratory equipment was calibrated, and blinded duplicate samples were analyzed. Data were collected by cardiologists and trained nurses using a standard questionnaire and an in-person interview during a single clinic visit. Before the survey was performed, we invited all eligible investigators to attend an organized training session. The training involved the purpose of this study, how to administer the questionnaire, the standard measurement methodology, the importance of standardization, and the study procedures. A strict test was conducted after this training, and only those who scored perfectly on the test were allowed to serve as investigators. During data collection, our inspectors provided further instructions and support.

\section{ECG Method}

Standard 12-lead ECGs were used with a MAC 5500 (GE Healthcare, Little Chalfont, UK), as previously described, ${ }^{21}$ and the ECGs were analyzed automatically with the MUSE Cardiology Information System, version 7.0.0 (GE Healthcare). ECG parameters, including heart rate (HR), QRS width, QT interval, PR interval (PR), and RR interval (RR), were measured automatically. QT interval was measured from the earliest detection of depolarization in any lead to the latest detection of repolarization in any lead. Because QT interval is influenced by heart rate, it was necessary to adjust QT interval for heart rate. ${ }^{22}$ In this study, the QTc interval corrected by heart rate was calculated with the Bazett, Fredericia, and Framingham formulas. $^{23-25}$

\section{Echocardiography Method}

Echocardiography methods were consistent with our previous study. ${ }^{21}$ Briefly, echocardiography was performed in all participants according to American Society of Echocardiography guidelines. A Doppler echocardiography (Vivid; GE Healthcare, Connecticut, USA) with a $3.0-\mathrm{MHz}$ transducer was used by the sonographers. Transthoracic echocardiography constituted M-mode, twodimensional, spectral, and color Doppler formats with subjects in the supine position. We took a parasternal acoustic window to record the systolic diameter and posterior wall thickness of left ventricular, as well as the M-mode images of the aortic root and left atrium. An apical acoustic window was used to record four-chamber and five-chamber images. The orientation of the imaging planes and Doppler recordings were verified according to previous procedures. Interventricular septal thickness (IVSd), posterior wall thickness (LVPW), and left ventricular internal dimensions were measured at the end of diastole (LVIDD); left ventricular internal dimensions were measured at the end of systole (LVIDS). ${ }^{26}$

LV ejection fraction (LVEF) was calculated using the modified Simpson's rules. ${ }^{27}$ Doppler echocardiographic recordings were performed with the sample volume at the tips of the mitral valve leaflets in the apical fourchamber view. Peak early diastolic filling wave velocity, peak atrial diastolic filling wave velocity, and deceleration time were measured. Aortic root diameter (ARD) was measured at the aortic annulus level in the parasternal long-axis view. Doppler waves of aortic blood flow were recorded in the apical five-chamber view or subcostal view. The aortic velocity-time integral was measured at end-expiration, and maximum peak aortic blood flow velocities (AV peak) were obtained during a single respiratory cycle. Echocardiography readings and analyses were performed by three medical doctors who are experts in this field. If questions or uncertainties arose, the option for consultation with two additional specialists was available.

\section{Adjudication of Endpoints}

In this study, we defined an incident CVD event as a composite of new onset stroke or coronary heart disease during the follow-up period. Specific incidences of stroke and coronary heart disease were also determined. All available clinical information was collected, including 
medical records and death certificates. All materials were independently reviewed and adjudicated by the endpoint assessment committee. Stroke was defined according to the World Health Organization (WHO) Multinational Monitoring of Trends and Determinants in CVD criteria as rapidly developing signs of focal or global disturbance of cerebral function lasting more than 24 hours (unless interrupted by surgery or death), with no apparent nonvascular cause. ${ }^{28}$ Hemorrhagic stroke was specifically defined as stroke with a diagnosis of subarachnoid hemorrhage or intracerebral hemorrhage, and ischemic stroke was defined as stroke with a diagnosis of thrombosis or embolism. Transient ischemic attack and chronic cerebrovascular disease were excluded. Coronary heart disease was defined as a diagnosis of hospitalized angina, hospitalized myocardial infarction, coronary heart disease death, or any revascularization procedure. ${ }^{29}$

\section{Data Selection and Grouping}

To evaluate all information available in this study for CVD occurrence, we assessed 76 variables (details are shown in Supplemental Table 1) from the patients' basic information, biochemical results, ECG results, and echocardiography results.

\section{Statistical Analysis}

Variable Screening and Model Construction

Least absolute shrinkage and selection operator (LASSO) regression is a method of latitude reduction for multidimensional data. ${ }^{30}$ We used LASSO regression to reduce the dimension of the current variables, then we used the selected variables to construct prognostic models (Model 1). Furthermore, we classified participants into low- and high-risk groups according to the CVD models. We then constructed Model 2 for comparison using COX regression with Framingham-related variables. ${ }^{31}$

\section{Evaluation of Clinical Usefulness, and Visualization}

Traditional diagnostic performance metrics, namely sensitivity, specificity, and area under the curve, can measure only the diagnostic accuracy of one predictive model relative to another, but cannot explain the clinical applicability of a specific model. Decision Curve Analysis (DCA) quantified net benefit under different thresholds in the dataset to evaluate clinical applicability. A nomogram is a tool that visualizes and scores a model. We created a nomogram to evaluate the model visually with a contour map. In addition, we used the C-index to evaluate the distinction between the primary and verification sets. Calibration diagrams and Hosmer-Lemeshow testing were used to evaluate the calibration of the model. In addition, we explored the potential nonlinear associations using triple-knotted restricted cubic spline regression to test the correlation between hazard ratios (HR) and model score.

\section{Compare Old Models with New Models}

We used the Net Reclassification Index (NRI) statistical method $^{32,33}$ to compare the old with new models according to the continuous variables. Briefly, the old diagnostic index classified the people into patients and non-patients, and the new diagnostic index would reclassify them. At this time, we found that some people who were originally misclassified in the old diagnostic indicators, were correctly classified in the new diagnostic indicators and vice versa. We used this reclassification change to calculate the net reclassification Index (NRI). Compared with the old model, the reclassification rate of the new model increased by $20 \%$ (95\% CI: $16.8-34.6 \%)$.

All data analysis was completed using R (version 3.6.1; http://www.Rproject.org). Missing values were filled using MICE packages. Descriptive statistics were calculated for all variables, namely continuous variables (reported as mean values and standard deviations if normally distributed, used nonparametric test if abnormally distributed) and categorical variables (reported as numbers and percentages). Differences between categories were evaluated using the $t$-test, analysis of variance (ANOVA), nonparametric test, or $\chi^{2}$-test as appropriate. LASSO regression was performed using glmnet packages and COX regression was performed using the MASS package. The CompareC package was used to compare the $\mathrm{C}$-index of two models; DCA analysis was based on rmda packages, and the contour map was visualized by rms. All of the statistical analyses were performed using SPSS version 20.0 software, and $\mathrm{p}$ values less than 0.05 were considered statistically significant.

\section{Results}

\section{Updated Incidence of CVD from the $\mathrm{NCRCHS}$}

The baseline characteristics of the included participants in the NCRCHS cohort were published in $2016 .{ }^{21}$ For the entire population completing the current follow-up study $(n=10,349)$, the median follow-up time was 4.66 years. After we excluded participants with a history of CVD at baseline ( $\mathrm{n}=821$ ), results indicated a total incidence density of CVD of 1.1\%/year (incidence of fatal CVD per year: $0.3 \%$; nonfatal CVD: $0.7 \%$ ), including stroke 
Table I Incidence of CVD in NCRCHS Cohort (Subjects with a Specific Disease History Were Excluded from the Baseline According to the Corresponding Outcome Events)

\begin{tabular}{|l|c|c|c|c|}
\hline Incidence & $\begin{array}{c}\text { CVD (\% } \\
\text { per } \\
\text { Year) }\end{array}$ & $\begin{array}{c}\text { Stroke } \\
\text { (\%per } \\
\text { Year) }\end{array}$ & $\begin{array}{c}\text { Coronary Heart } \\
\text { Disease (\%per } \\
\text { Year) }\end{array}$ & $\begin{array}{c}\text { Myocardial } \\
\text { Infarction (\% } \\
\text { per Year) }\end{array}$ \\
\hline Total & $470(1.1)$ & $342(0.7)$ & $175(0.4)$ & $80(0.2)$ \\
Fatal & $161(0.3)$ & $109(0.2)$ & $68(0.1)$ & $53(0.1)$ \\
Nonfatal & $309(0.7)$ & $233(0.5)$ & $107(0.2)$ & $27(0.1)$ \\
\hline
\end{tabular}

Abbreviation: CVD, cardiovascular disease (including stroke and coronary hear disease).

$(n=342)$ and coronary heart disease $(n=175)$. The specific incidences of stroke and coronary heart disease are shown in Table 1. The patient's flow is shown in Figure 1.

\section{Risk Factors for CVD from the Predictive Model}

To assess the impact of demographic characteristics, biochemical results, ECG results, and echocardiography results on CVD occurrence, we divided the study group into a primary cohort and a validation cohort according to region. Complete information on co-variables from the baseline visit was required for inclusion in this analysis, and sufficient data was available for 6606 participants in the primary cohort (training data) and 2918 in the validation cohort (external validation) (Figure 2). There was no significant difference in CVD incidence between the cohorts: $1.1 \%$ /year in the primary cohort and $1.3 \%$ / year in the validation cohort $(P=0.39)$. Participant characteristics for both cohorts are given in Supplemental Table 2.

We constructed Model 1 using the variables selected by LASSO according to data for 313 CVD patients in the primary cohort. From all information, namely demographic characteristics, blood biochemical indicators, ECG characteristics, and echocardiography indicators, 70 factors for each participant were reduced to 23 potential predictors with nonzero coefficients in the LASSO regression model, including age, gender, and LDL-C, and others. We first established the minimum lambda value of 0.0030 through LASSO regression analysis, then used this value to filter the variables (Supplemental Figure 1 and Table 2).

The influence of the factors on CVD occurrence are seen in Figure 3 and Supplemental Table 2 (Model 1). As evident from the results, age, smoking, diabetes, FPG, waist circumference, blood pressure, LDL, BUN, SCr, eGFR, and a family history of stroke were CVD risk factors. In addition to the above, QTc (Framingham) hazard radio (HR): 1.013; 95\% confidence interval (CI: 1.010-1.016; $p<0.001)$, ARD (HR: 1.462; 95\% CI: 1.223-1.748; $p<0.001$ ), and IVSd (HR:

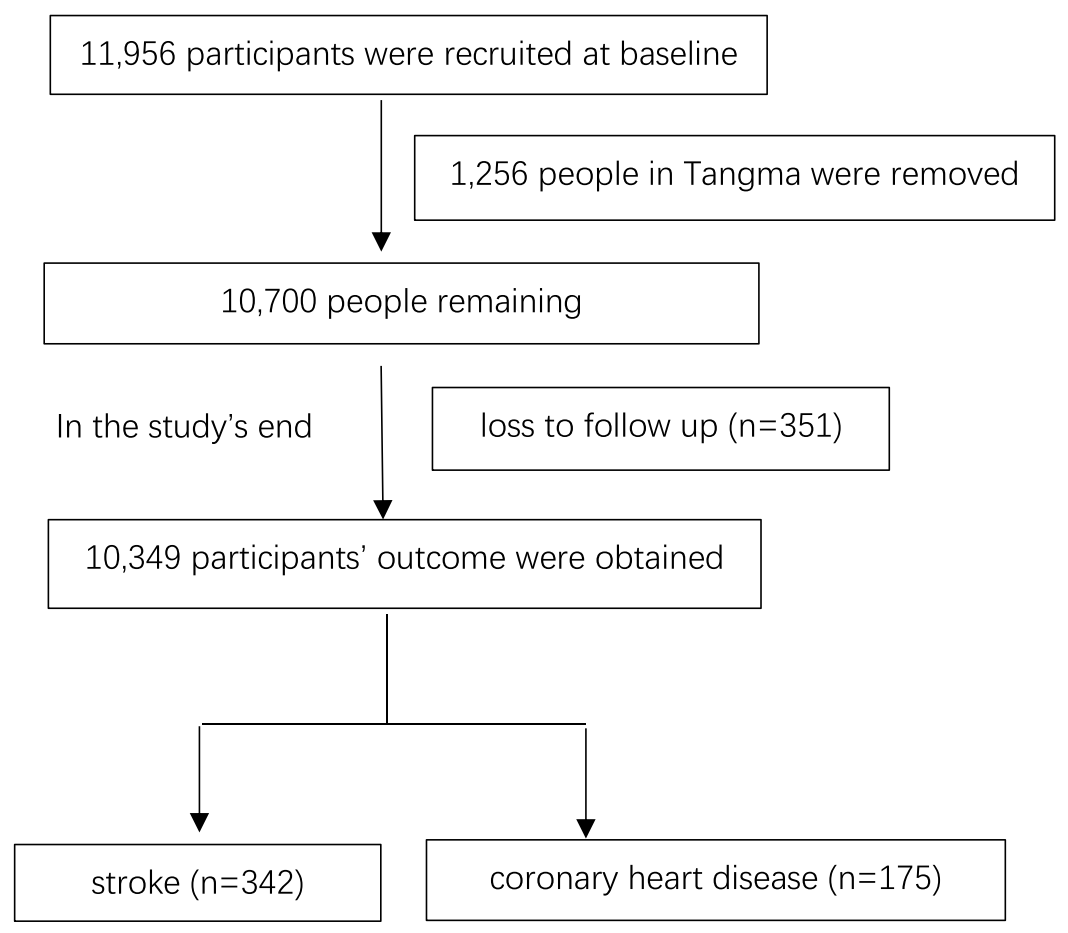

Figure I Patient's flow. 


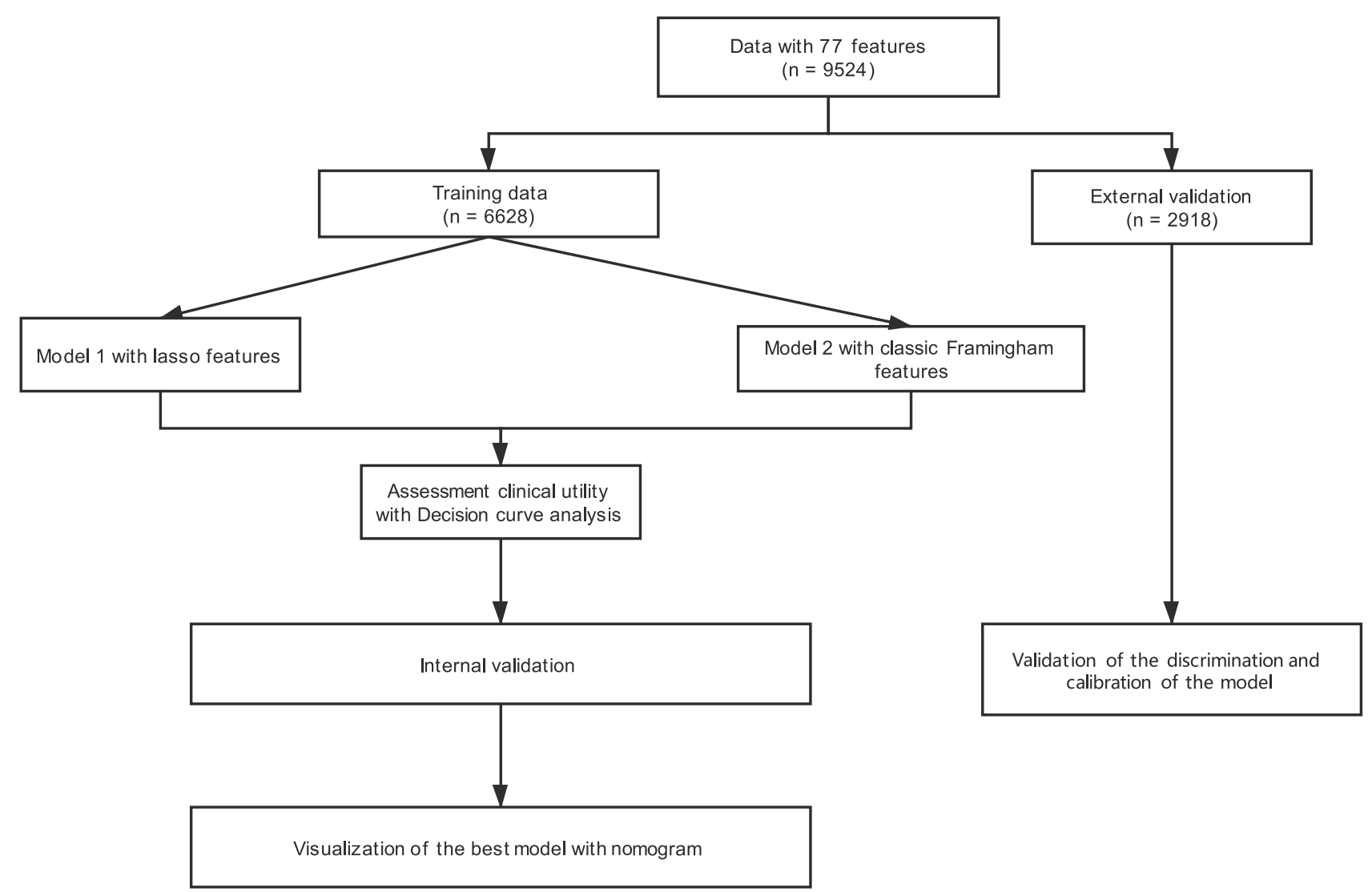

Figure 2 Study flowchart. LASSO, least absolute shrinkage and selector operation. The model's discrimination was validated with the C-index. The model's calibration was validated with a calibration plot and the Hosmer-Lemeshow test.

1.487; 95\% CI: $1.287-1.717 ; p<0.001)$ were also predictive factors for CVD.

\section{Evaluation and Validation of the Predictive Model}

Framingham-related variables, namely age, sex, smoking, TC, HDL-C, and diabetes status were used to construct Model 2 using Cox regression. A DCA curve was then used to compare the clinical practicability of the two models. As Figure 4A shows, the net benefit of Model 1 was higher than that of Model 2, $\mathrm{P}=0.0053$.

Moreover, a linear and positive association between the model total score and the risk of incident CVD events using restricted cubic spline regression was also seen (for nonlinearity, $\mathrm{P}=0.617$ ) (Figure $4 \mathrm{~B}$ ).

Model 1 was validated in both the primary and verification cohorts, with a C-index of 0.785 (95\% CI: 0.784-0.786) and 0.749 (95\% CI: $0.748-0.751)$. We then evaluated whether there was a difference in the $\mathrm{C}$-index of the two models. Statistical analysis showed that the $\mathrm{C}$-index of the two models differed significantly $(\mathrm{P}=0.0024)$. We also verified the calibration of the model. As shown in Figure 5, the validation and primary sets were comparatively consistent. In addition, the Hosmer-Lemeshow test yielded nonsignificant results: $\mathrm{P}=1$ (validation sets) and $\mathrm{P}=1$ (primary sets).

We used our model to group the test set with high and low risks. After analysis, we found that out of 2918 participants, 1344 were qualified as high-risk while 1574 belonged to the low-risk group (Supplemental Table 3).

Finally, we used the Net Reclassification Index (NRI) statistical method to compare the old and new models according to the continuous variables. Compared with the old model, the reclassification rate of the new model increased by $20 \%$ (95\% CI: $16.8-34.6 \%$ ).

\section{Visualization of the Predictive Model}

To predict the possibility of CVD in 2 or 4 years, we created a nomogram to visualize Model 1. As shown in Figure 6, we used Cox regression to measure the values of the variables and identify the corresponding position of each variable on the nomogram. The total score of the value was obtained by adding the individual scores. 
Table 2 Participant Characteristics in the Primary and Validation Cohorts

\begin{tabular}{|c|c|c|c|c|c|c|}
\hline \multirow[t]{3}{*}{ Variables } & Primary Data Set & & & Validation Data Set & & \\
\hline & CVD & & & CVD & & \\
\hline & No & Yes & $\mathbf{p}$ & No & Yes & $\mathbf{p}$ \\
\hline $\mathrm{n}$ & 6293 & 313 & & 2762 & 156 & \\
\hline Age (years) & $53.1 \pm 10.1$ & $62.2 \pm 9.7$ & $<0.001$ & $52.0 \pm 10.5$ & $60.1 \pm 9.3$ & $<0.001$ \\
\hline Family_income (CNY/year) & $25,101 \pm 25,808$ & $20,522 \pm 24,673$ & 0.002 & $18,983 \pm 21,358$ & $19,404 \pm 34,480$ & 0.818 \\
\hline Medical_expense (CNY/year) & $4082 \pm \mid 1,321$ & $4533 \pm 8759$ & 0.487 & $5846 \pm|5,73|$ & $6279 \pm 11,583$ & 0.734 \\
\hline Sleep_duration (hours) & $7.2 \pm 1.7$ & $7.0 \pm 1.8$ & 0.036 & $7.7 \pm 1.6$ & $7.5 \pm 1.8$ & 0.302 \\
\hline $\mathrm{BMI}\left(\mathrm{kg} / \mathrm{m}^{2}\right)$ & $24.5 \pm 3.5$ & $24.9 \pm 3.5$ & 0.068 & $25.3 \pm 3.8$ & $26.2 \pm 3.6$ & 0.007 \\
\hline Mean_TW (cm) & $95.1 \pm 6.8$ & $95.1 \pm 7.3$ & 0.981 & $97.5 \pm 7.5$ & $98.5 \pm 8.6$ & 0.107 \\
\hline Mean_SBP (mmHg) & $137.3 \pm 20.8$ & $152.8 \pm 25.5$ & $<0.001$ & $147.2 \pm 23.8$ & $168.1 \pm 28.5$ & $<0.001$ \\
\hline Mean_DBP (mmHg) & $81.6 \pm 11.3$ & $86.3 \pm \mid 4.1$ & $<0.001$ & $81.8 \pm 11.7$ & $88.6 \pm 15.8$ & $<0.001$ \\
\hline Mean_pulse (bpm) & $80.1 \pm 13.3$ & $80.3 \pm 14.4$ & 0.88 & $73.6 \pm 11.4$ & $76.0 \pm 12.3$ & 0.012 \\
\hline Aortic root diameter $(\mathrm{cm})$ & $2.2 \pm 0.3$ & $2.3 \pm 0.4$ & $<0.001$ & $2.3 \pm 0.4$ & $2.3 \pm 0.3$ & 0.203 \\
\hline Interventricular septal thickness $(\mathrm{cm})$ & $0.9 \pm 0.2$ & $0.9 \pm 0.2$ & $<0.001$ & $0.9 \pm 0.2$ & $0.9 \pm 0.1$ & 0.003 \\
\hline End-diastolic diameter of left ventricle $(\mathrm{cm})$ & $4.7 \pm 0.4$ & $4.8 \pm 0.5$ & 0.010 & $4.7 \pm 0.4$ & $4.8 \pm 0.6$ & 0.003 \\
\hline Left ventricular end systolic diameter $(\mathrm{cm})$ & $3.1 \pm 0.4$ & $3.2 \pm 0.5$ & 0.025 & $3.1 \pm 0.5$ & $3.3 \pm 0.7$ & $<0.001$ \\
\hline Left ventricular posterior wall thickness $(\mathrm{cm})$ & $0.9 \pm 0.3$ & $0.9 \pm 0.2$ & 0.008 & $0.9 \pm 0.3$ & $1.0 \pm 0.6$ & $<0.001$ \\
\hline Left ventricular ejection fraction (\%) & $63.3 \pm 3.7$ & $62.2 \pm 4.5$ & $<0.001$ & $62.2 \pm 3.4$ & $60.7 \pm 4.0$ & $<0.001$ \\
\hline Mitral valve $\mathrm{E}$ peak velocity $(\mathrm{cm} / \mathrm{s})$ & $73.0 \pm 20.9$ & $66.8 \pm 20.7$ & $<0.001$ & $78.1 \pm 35.9$ & $71.1 \pm 20.9$ & 0.017 \\
\hline Mitral valve $A$ peak velocity $(\mathrm{cm} / \mathrm{s})$ & $76.4 \pm 23.7$ & $83.3 \pm 20.5$ & $<0.001$ & $74.3 \pm 18.0$ & $83.0 \pm 17.9$ & $<0.001$ \\
\hline Aortic valve velocity $(\mathrm{cm} / \mathrm{s})$ & $120.8 \pm 22.6$ & $122.8 \pm 27.6$ & 0.133 & $117.1 \pm 19.3$ & $117.7 \pm 23.78$ & 0.684 \\
\hline Interventricular septal hypertrophy $(\mathrm{cm})$ & $0.02 \pm 0.2$ & $0.1 \pm 0.3$ & $<0.001$ & $0.02 \pm 0.1$ & $0.06 \pm 0.3$ & 0.001 \\
\hline WBC $\left(10^{9} / \mathrm{L}\right)$ & $6.1 \pm 2.0$ & $6.3 \pm 2.0$ & 0.191 & $6.2 \pm 1.6$ & $6.2 \pm 1.7$ & 0.927 \\
\hline $\mathrm{Hb}(\mathrm{g} / \mathrm{L})$ & $136.2 \pm 16.6$ & $136.9 \pm 15.5$ & 0.492 & $144.4 \pm 15.7$ & $144.6 \pm 20.1$ & 0.879 \\
\hline $\operatorname{RBC}\left(10^{12} / \mathrm{L}\right)$ & $4.6 \pm 0.5$ & $4.6 \pm 0.5$ & 0.765 & $4.6 \pm 0.4$ & $4.7 \pm 0.5$ & 0.533 \\
\hline $\mathrm{HCT}(\%)$ & $36.1 \pm 15.3$ & $37.8 \pm 13.7$ & 0.053 & $42.5 \pm 12.4$ & $42.4 \pm 4.2$ & 0.894 \\
\hline $\mathrm{MCV}(\mathrm{fL})$ & $96.7 \pm 66.6$ & $94.7 \pm 45.6$ & 0.599 & $97.5 \pm 237.9$ & $91.4 \pm 5.4$ & 0.752 \\
\hline $\mathrm{MCH}(\mathrm{pg})$ & $29.7 \pm 2.8$ & $29.7 \pm 3.1$ & 0.983 & $31.2 \pm 2.5$ & $31.2 \pm 2.5$ & 0.974 \\
\hline $\mathrm{MCHC}(g / \mathrm{L})$ & $325.2 \pm 23.4$ & $324.4 \pm 19.2$ & 0.540 & $344.3 \pm 83.4$ & $341.4 \pm 12.3$ & 0.663 \\
\hline $\operatorname{PLT}\left(10^{9} / \mathrm{L}\right)$ & $208.7 \pm 60.8$ & $203.2 \pm 68.9$ & 0.124 & $230.7 \pm 57.1$ & $224.0 \pm 56.3$ & 0.154 \\
\hline MPV (fL) & $10.6 \pm 2.3$ & $10.4 \pm 2.0$ & 0.334 & $11.4 \pm 2.8$ & $11.4 \pm 0.8$ & 0.980 \\
\hline PDW (\%) & $15.5 \pm 1.9$ & $15.6 \pm 1.6$ & 0.476 & $13.4 \pm 4.8$ & $13.3 \pm 2.0$ & 0.718 \\
\hline РCT (\%) & $0.2 \pm 0.1$ & $0.2 \pm 0.1$ & 0.041 & $0.3 \pm 0.2$ & $0.3 \pm 0.1$ & 0.482 \\
\hline ALT (u/L) & $22.5 \pm 16.6$ & $21.9 \pm 15.1$ & 0.586 & $22.4 \pm 15.8$ & $25.3 \pm 25.5$ & 0.035 \\
\hline AST $(u / L)$ & $21.4 \pm 12.2$ & $22.1 \pm 13.7$ & 0.303 & $24.2 \pm 14.0$ & $27.1 \pm 16.2$ & 0.015 \\
\hline AST_AL & $I . I \pm 0.5$ & $1.2 \pm 0.6$ & 0.030 & $1.2 \pm 0.6$ & $1.3 \pm 0.6$ & 0.185 \\
\hline BUN (mmol/L) & $5.5 \pm 2.4$ & $5.7 \pm 1.6$ & 0.168 & $5.7 \pm 1.6$ & $6.0 \pm 1.8$ & 0.012 \\
\hline $\mathrm{SCr}$ (umol/L) & $74.8 \pm 15.1$ & $81.9 \pm 44.0$ & $<0.001$ & $62.1 \pm 16.3$ & $65.5 \pm 17.3$ & 0.011 \\
\hline eGFR (mL/min) & $90.6 \pm 14.3$ & $81.1 \pm 13.6$ & $<0.001$ & $103.7 \pm 12.2$ & $95.8 \pm 13.9$ & $<0.001$ \\
\hline UA (umol/L) & $295.6 \pm 82.4$ & $314.0 \pm 88.6$ & $<0.001$ & $266.9 \pm 77.9$ & $279.4 \pm 80.1$ & 0.051 \\
\hline GLU (mmol/L) & $5.9 \pm 1.4$ & $6.3 \pm 2.0$ & $<0.001$ & $5.7 \pm 1.82$ & $6.09 \pm 1.53$ & 0.021 \\
\hline $\mathrm{TCH}(\mathrm{mmol} / \mathrm{L})$ & $5.3 \pm 1.1$ & $5.6 \pm 1.1$ & $<0.001$ & $5.0 \pm 1.0$ & $5.3 \pm 1.0$ & $<0.001$ \\
\hline $\mathrm{TG}(\mathrm{mmol} / \mathrm{L})$ & $1.6 \pm 1.4$ & $1.7 \pm 1.1$ & 0.202 & $1.5 \pm 1.5$ & $1.7 \pm 1.9$ & 0.139 \\
\hline HDL_C (mmol/L) & $1.4 \pm 0.3$ & $1.3 \pm 0.3$ & 0.074 & $1.6 \pm 0.5$ & $1.6 \pm 0.5$ & 0.671 \\
\hline LDL_C $(\mathrm{mmol} / \mathrm{L})$ & $2.9 \pm 0.8$ & $3.1 \pm 0.8$ & $<0.001$ & $3.0 \pm 0.8$ & $3.24 \pm 0.92$ & $<0.001$ \\
\hline $\mathrm{Ca}(\mathrm{mmol} / \mathrm{L})$ & $2.3 \pm 0.1$ & $2.3 \pm 0.1$ & 0.492 & $2.4 \pm 0.1$ & $2.4 \pm 0.1$ & 0.484 \\
\hline $\mathrm{P}(\mathrm{mmol} / \mathrm{L})$ & $I . I \pm 0.2$ & $1.1 \pm 0.2$ & 0.140 & $1.1 \pm 0.2$ & $1.1 \pm 0.2$ & 0.864 \\
\hline $\mathrm{Mg}(\mathrm{mmol} / \mathrm{L})$ & $0.8 \pm 0.1$ & $0.8 \pm 0.1$ & 0.215 & $0.9 \pm 0.1$ & $0.9 \pm 0.1$ & 0.791 \\
\hline $\mathrm{K}(\mathrm{mmol} / \mathrm{L})$ & $4.2 \pm 0.3$ & $4.2 \pm 0.4$ & 0.851 & $4.2 \pm 0.4$ & $4.2 \pm 0.4$ & 0.632 \\
\hline $\mathrm{SNa}(\mathrm{mmol} / \mathrm{L})$ & $141.3 \pm 1.8$ & $141.6 \pm 1.7$ & 0.001 & $140.8 \pm 2.9$ & $|4| .2 \pm 3.0$ & 0.085 \\
\hline $\mathrm{SCl}(\mathrm{mmol} / \mathrm{L})$ & $101.5 \pm 2.2$ & $101.8 \pm 2.3$ & 0.025 & $103.8 \pm 2.5$ & $103.7 \pm 2.8$ & 0.926 \\
\hline PR (ms) & $151.4 \pm 22.8$ & $153.2 \pm 28.5$ & 0.189 & $151.2 \pm 22.3$ & $150.7 \pm 26.1$ & 0.792 \\
\hline PRms & $416.3 \pm 20.7$ & $423.3 \pm 21.0$ & $<0.001$ & $152.1 \pm 20.7$ & $152.0 \pm 20.5$ & 0.952 \\
\hline
\end{tabular}


Table 2 (Continued).

\begin{tabular}{|c|c|c|c|c|c|c|}
\hline \multirow[t]{3}{*}{ Variables } & Primary Data Set & & & Validation Data Set & & \\
\hline & CVD & & & CVD & & \\
\hline & No & Yes & $\mathbf{p}$ & No & Yes & $\mathbf{p}$ \\
\hline QTcFramingham (ms) & $415.6 \pm 22.0$ & $422.9 \pm 21.9$ & $<0.001$ & $421.9 \pm 21.4$ & $428.8 \pm 22.3$ & $<0.001$ \\
\hline QTcFredericia (ms) & $428.1 \pm 24.9$ & $435.9 \pm 23.9$ & $<0.001$ & $421.4 \pm 22.1$ & $428.5 \pm 23.5$ & $<0.001$ \\
\hline QTcBazett (ms) & $12.0 \pm 2.1$ & $12.1 \pm 2.2$ & 0.462 & $430.1 \pm 25.3$ & $440.6 \pm 24.9$ & $<0.001$ \\
\hline QRS (ms) & $843.7 \pm 137.7$ & $841.6 \pm 142.8$ & 0.788 & $11.4 \pm 1.9$ & $11.9 \pm 2.2$ & $<0.001$ \\
\hline $\mathrm{RR}(\mathrm{ms})$ & $81.2 \pm 9.6$ & $83.5 \pm 9.3$ & $<0.001$ & $890.3 \pm 137.9$ & $850.1 \pm 140.0$ & $<0.001$ \\
\hline Mean_WC (cm) & $81.9 \pm 9.7$ & $84.7 \pm 10.2$ & $<0.001$ & $83.5 \pm 9.8$ & $87.1 \pm 11.4$ & $<0.001$ \\
\hline Gender (male) & $3416(54.3 \%)$ & 143 (45.7\%) & 0.004 & 1339 (48.5\%) & $86(55.1 \%)$ & 0.125 \\
\hline Marriage (married) & $5814(92.4 \%)$ & $265(84.7 \%)$ & $<0.001$ & 2504 (90.8\%) & 127 (81.4\%) & $<0.001$ \\
\hline Race (Mongolian) & 145 (2.3\%) & II (3.5\%) & 0.236 & 221 (8.0\%) & $14(9.0 \%)$ & 0.777 \\
\hline Kidney disease & $123(2.0 \%)$ & $\mathrm{I}(0.3 \%)$ & 0.062 & $132(4.8 \%)$ & $6(3.8 \%)$ & 0.734 \\
\hline DM & 242 (3.8\%) & $29(9.3 \%)$ & $<0.001$ & $68(2.5 \%)$ & $6(3.8 \%)$ & 0.419 \\
\hline Family_DM & $968(15.4 \%)$ & 49 (I5.7\%) & 0.960 & $220(1 \%)$ & $16(10.3 \%)$ & 0.384 \\
\hline Family_HT & 1232 (19.6\%) & $69(22.0 \%)$ & 0.318 & $783(28.3 \%)$ & $48(30.8 \%)$ & 0.575 \\
\hline Family_CHD & $818(13.0 \%)$ & 35 (11.2\%) & 0.396 & $436(15.1 \%)$ & $19(12.2 \%)$ & 0.274 \\
\hline Family_stroke & 833 (13.2\%) & 57 (I8.2\%) & 0.015 & 614 (22.2\%) & 34 (21.8\%) & 0.977 \\
\hline Family_AF & 458 (7.3\%) & 28 (8.9\%) & 0.321 & $17(0.6 \%)$ & I (0.6\%) & 1.000 \\
\hline Current_smoke & 2124 (33.8\%) & $130(4 \mid .5 \%)$ & 0.006 & 1096 (39.4\%) & $65(41.7 \%)$ & 0.683 \\
\hline Current_drink & 1380 (2I.9\%) & 69 (22.0\%) & 1.000 & 757 (27.3\%) & 47 (30.1\%) & 0.517 \\
\hline Snoring & 2444 (38.8\%) & $|3|(4 \mid .9 \%)$ & 0.313 & 992 (35.4\%) & $67(42.9 \%)$ & 0.091 \\
\hline Degenerative & $1192(18.9 \%)$ & 94 (30.0\%) & $<0.001$ & 969 (35.5\%) & $80(51.3 \%)$ & $<0.001$ \\
\hline Long QTC & 487 (7.7\%) & 45 (14.4\%) & $<0.001$ & $267(9.7)$ & $35(22.4)$ & $<0.001$ \\
\hline
\end{tabular}

Abbreviations: CNY, China Yuan (ICNY = 0.1542 USD); bpm, beat per minute; CVD, cardiovascular disease; Family_CHD, participant with immediate family member who has coronary atherosclerotic heart disease; Family_DM, participant with immediate family member who has diabetes mellitus; Family_HT, participant with immediate family member who has hypertension; Family_stroke, participant with immediate family member who has stroke; Family_AF, participant with immediate family member who has atrial fibrillation; BMI, body mass index; TW, hip circumference; WC, waist circumference; SBP, systolic blood pressure; DBP, diastolic blood pressure; FPG, fasting plasma glucose; LDL-C, low-density lipoprotein cholesterol; HDL-C, high density lipoprotein cholesterol; eGFR, estimated glomerular filtration rate; Hgb, hemoglobin; BUN, blood urea nitrogen; $\mathrm{SCr}$, serum creatinine; $\mathrm{MCH}$, mean concentration of hemoglobin; $\mathrm{AST}$, aspartate aminotransferase; $\mathrm{SCl}$, serum chlorine; $\mathrm{SNa}$, serum sodium; $\mathrm{HCT}$, hematocrit; TC, total cholesterol; TG, triglyceride; HR, heart rate; QRS, the width of QRS; PR, PR interval; RR, RR interval; QT, QT interval.

Finally, the total score was be used to visualize the probability of CVD in the next 2 or 4 years.

\section{Analysis of Long QTc}

Long QTc was defined according to our previous research. ${ }^{34}$ After analysis, we found that there were significant differences of long QTc in both primary data set and validation data set (Table 2). Then the relationship between long QTc and CVD was also analyzed, HR: 2.001 (95\% CI 1.4592.744). $\mathrm{P}=1.68 \mathrm{e}-5$. We put this variable in the Model 1 for analysis. However, it was removed by lasso regression maybe because collinear with other variables.

\section{Discussion}

In the past NCRCHS cohort, we discovered the relationship between alcohol consumption and cardiovascular diseases,${ }^{35}$ metabolism was associated with ischemic stroke, ${ }^{36}$ diagnosed diabetes was associated with depression and so on. ${ }^{37}$ In this study, we updated the CVD incidence in the NCRCHS cohort. Based on this general population, we developed and validated a novel predictive model for CVD. We found that QT interval from electrocardiography, and aortic root diameter and ventricular septal thickness from echocardiography should be included in the CVD risk prediction models.

Compared with the classic CVD prediction model, for pooled cohort equations that are used mostly for White and African American men and women, ${ }^{38}$ the model we built is based mainly on data for Asian people. In addition, regarding the included indicators, we used more clinical indicators like electrocardiography and echocardiography, which were feasible since the costs of the examinations was inexpensive and these examinations were widespread and convenient. Furthermore, we used the NRI statistical method $^{39}$ to compare the Framingham and our new models according to continuous variables. The reclassification rate 


\section{Lasso regression for CVD}

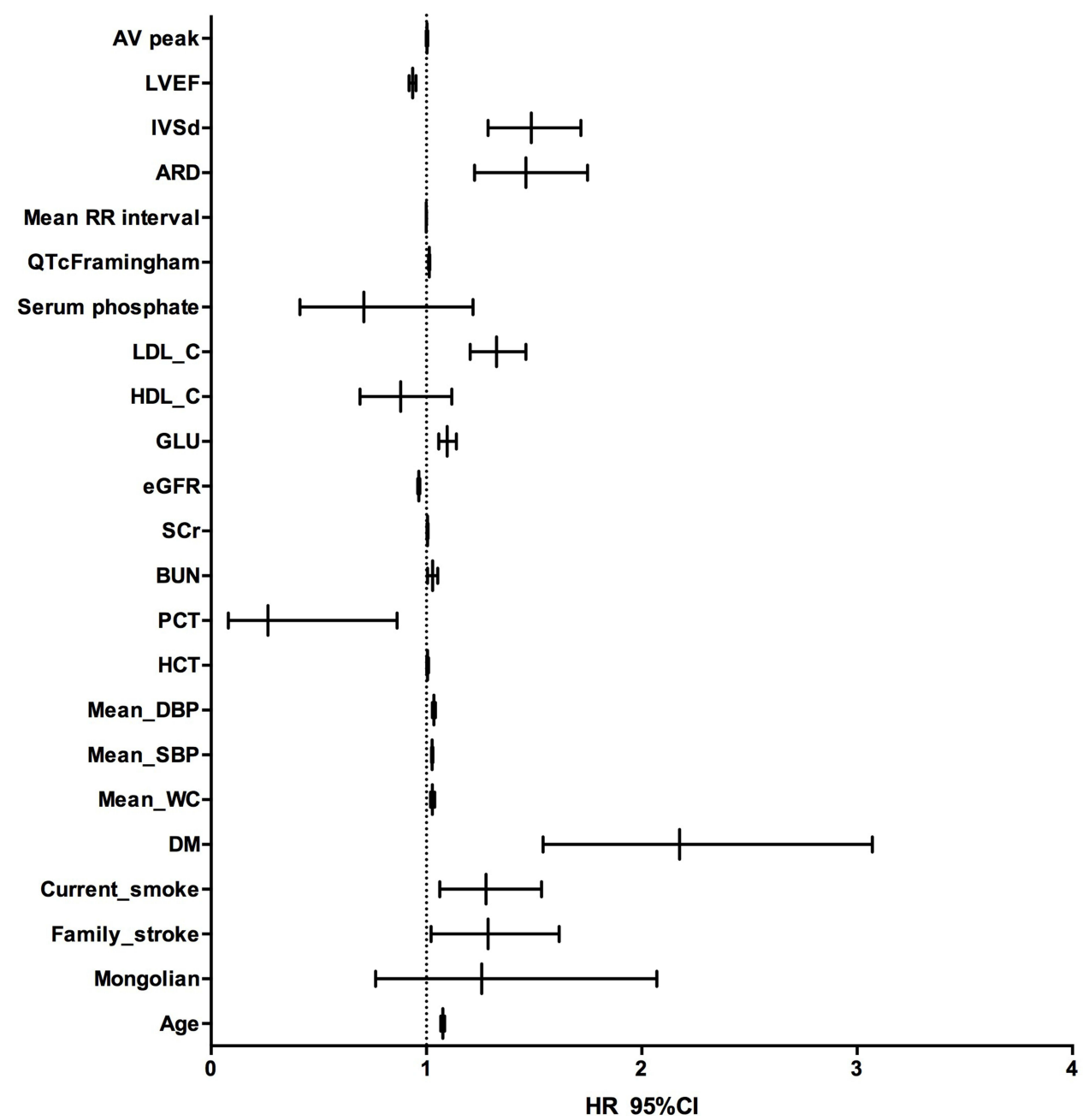

Figure 3 Influence of the factors screened by the LASSO regression on CVD occurrence.

Abbreviations: LASSO, least absolute shrinkage and selector operation; Family stroke, participant with an immediate family member who has experienced stroke; SBP, systolic blood pressure; DBP, diastolic blood pressure; FPG, fasting plasma glucose; LDL-C, low-density lipoprotein cholesterol; HDL-C, high-density lipoprotein cholesterol; eGFR, estimated glomerular filtration rate; Hgb, hemoglobin; BUN, blood urea nitrogen; SCr, serum creatinine; HCT, hematocrit; QTc Framingham, corrected QT interval calculated by the Framingham formula; LVEF, left ventricular ejection fraction; AV peak, maximum peak aortic blood flow velocity.

of the new model was increased by $20 \%$ (95\% CI: $16.8-$ $34.6 \%$ ) as compared to the old model. We believe that our model is optimized compared with classical models, especially when applied to Chinese populations.

In the current study, we identified novel risk factors for CVD from laboratory examinations, namely ECGs and echocardiography. In the general population, Dekker et $\mathrm{al}^{40}$ reported that QT prolongation was associated with an increased danger of coronary heart disease (CHD)- and CVD-related mortality in Black and White healthy men and women. In an Asian population in Pima, India, Kim et $\mathrm{al}^{41}$ discovered that prolonged QTc measurements and high heart rate both forecast all-cause mortality; however, heart rate was the better predictor. Maebuchi et $\mathrm{al}^{42}$ found that long QTc measurements were connected with stroke progress and CHD among Japanese men. The potential mechanism between long QTc and CVD is unidentified, but it has been reported to be associated with autonomic system dysfunction, ${ }^{43}$ genetic mutation of cardiac ion channels involved in cardiac repolarization, ${ }^{44}$ scar tissue after myocardial infarction, ${ }^{45}$ elevated blood sugar concentration, $^{46,47}$ high insulin level, ${ }^{47-49}$ hypokalemia, ${ }^{49}$ obesity, ${ }^{50}$ and ventricular hypertrophy. ${ }^{51}$ Standard echocardiography can be used to evaluate myocardial function, cardiac hemodynamics, valvular heart disease, and congenital heart disease. ${ }^{52}$ Utsunomiya et $\mathrm{al}^{53}$ revealed that 
A

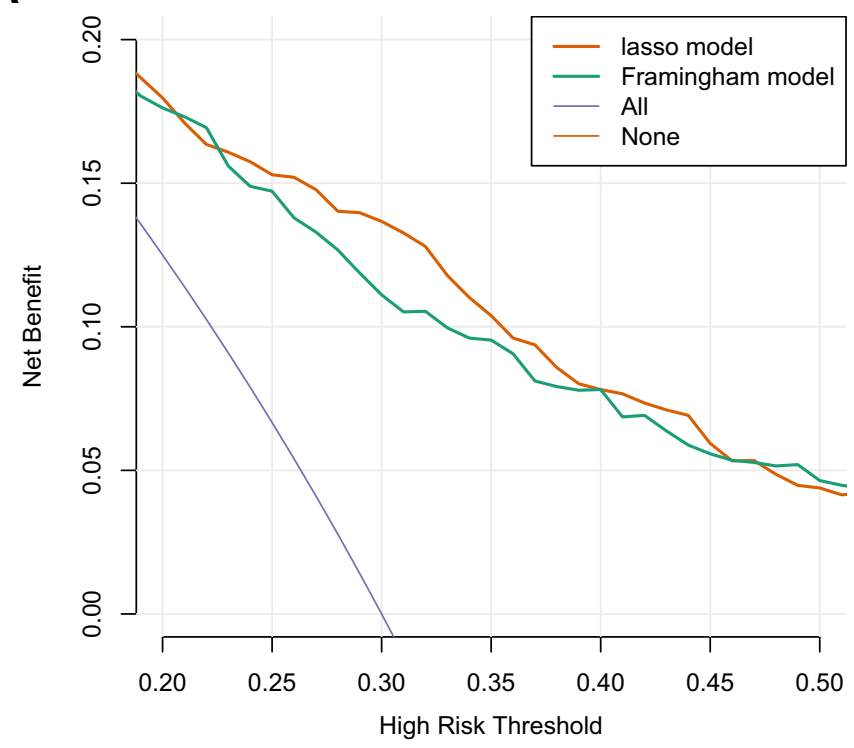

B

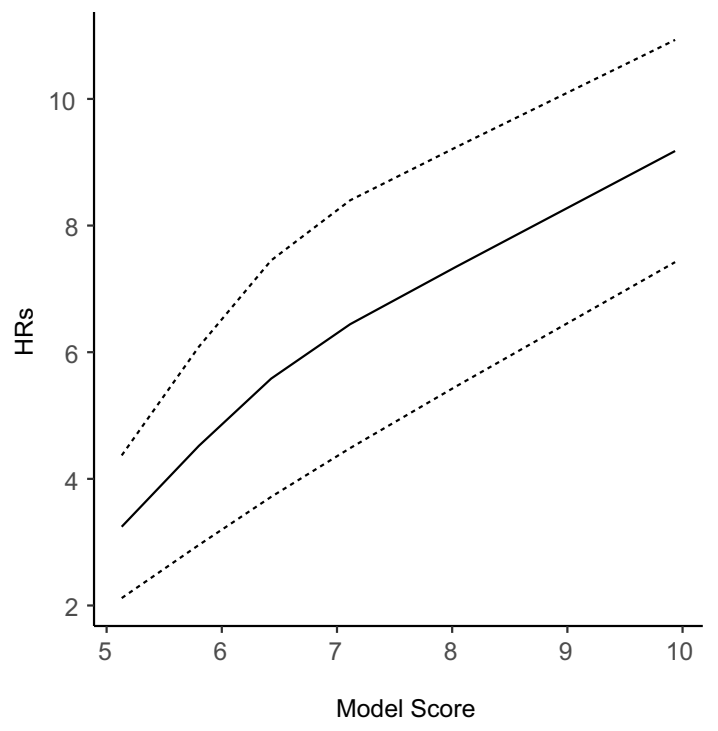

Figure 4 Evaluation of the models. (A) Decision curve analysis of the two-candidate model. The y-axis represents the net benefit. Model I was built with LASSO-selected features; Model 2 is the Framingham classic feature model. (B) Linear and positive association between the model total score and the risk of incident CVD events using restricted cubic spline regression (for nonlinearity, $\mathrm{P}=0.617$ ).

A

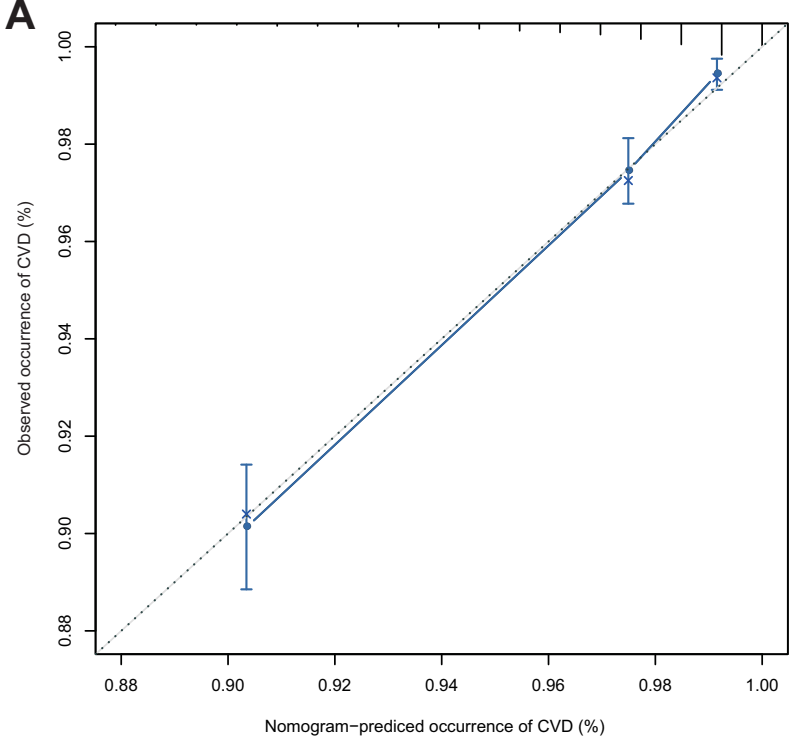

B

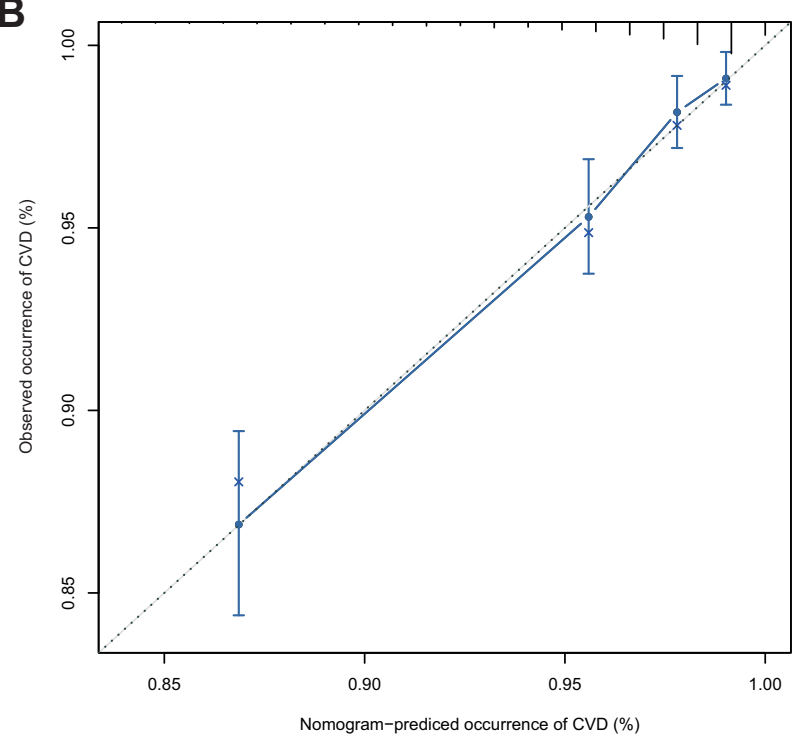

Figure 5 Calibration curves of the nomogram for the two cohorts. (A) Calibration curves in the primary cohort. (B) Calibration curves in the validation cohort.

echocardiographic total heart calcification (THC) score, which was composed of aortic valve calcification, mitral annular calcification, and aortic root calcification, had an enhancing and independent role in forecasting the mortality and morbidity of CVD. Liu et $\mathrm{al}^{54}$ showed that aortic root diameter (AoD) could be a useful marker to identify cardiovascular risk and heart failure (HF). The authors found that advanced age, and worse hypertension and diastolic blood pressure were associated with greater AoD. Hoang et $\mathrm{al}^{55}$ reported that left ventricular mass was related to CVD risk in older population with and without metabolic syndrome. Lin et $\mathrm{al}^{56}$ proved that left ventricular hypertrophy was a usual complication of hypertension. Park et $\mathrm{al}^{57}$ found that in nonhypertensive 

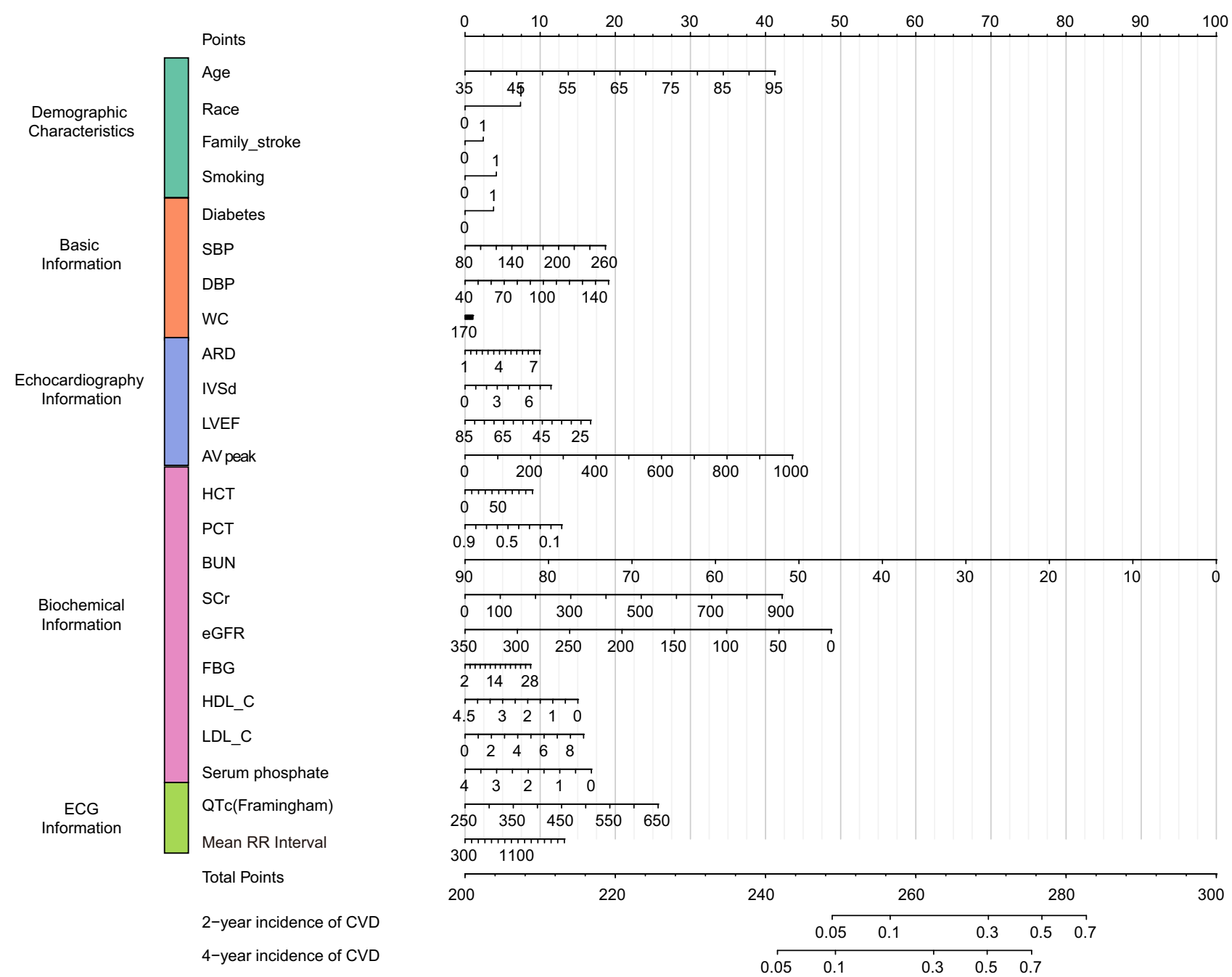

Figure 6 Nomogram for the best model. Family stroke = participant with immediate family member who has experienced stroke.

Abbreviations: SBP, systolic blood pressure; DBP, diastolic blood pressure; FPG, fasting plasma glucose; LDL-C, low-density lipoprotein cholesterol; HDL-C, high-density lipoprotein cholesterol; eGFR, estimated glomerular filtration rate; Hgb, hemoglobin; BUN, blood urea nitrogen; SCr, serum creatinine; HCT, hematocrit; QTc Framingham, corrected QT interval calculated by the Framingham formula; LVEF, left ventricular ejection fraction; AV peak, maximum peak aortic blood flow velocity.

populations, left ventricular geometry change, including left ventricular mass index, relative wall thickness, interventricular septal thickness (IVST), posterior wall thickness (PWT), and IVST plus PWT were closely related to an increased risk of hypertension. Loncaric et $\mathrm{al}^{58}$ revealed that basal ventricular septal hypertrophy could be a prognostic marker of the heavy load of hypertensive heart disease.

The findings of current studies have significant implications for public health policy. Currently, both doctors and patients rely heavily on traditional cardiovascular risk factors to evaluate potential CVD, which could lead to inadequate assessment. We found that QTc (Framingham), ARD, and IVSd were independent predictive risk factors for CVD in the general population. It is possible that patients with pathological echocardiography and ECGs represent patients with more advanced CVD than those without, which may be more likely to result in CVD in the short term. With the popularization of electrocardiography and echocardiography, we have more chances to identify patients who have potential risk factors and provide early intervention.

First, to our knowledge, this large-scale, communitybased study is the first to evaluate CVD risk by LASSO and to integrate demographic characteristics, blood biochemical indicators, ECG characteristics, and echocardiography indicators in a general cohort. A total of 10,349 among 11,956 participants $(86.6 \%)$ completed at least one 
follow-up visit; therefore, loss to follow-up may have slightly impacted our results. Our study further offers evidence to confirm that $\mathrm{BUN}, \mathrm{SCr}$, and eGFR are risk factors for CVD, as reported in recent studies. ${ }^{59}$ We also obtained consistent results indicating that the traditional factors of age, smoking, WC, SBP, DBP, FPG, LDL-C, and family history are indeed risk factors for CVD. Second, we used $\mathrm{DCA}^{60}$ to evaluate whether a diagnostic method is clinically useful. It is well known that many authors use receiver operating characteristic (ROC) curves to calculate areas under the curves (AUCs). However, ROC curve analysis focuses only on the specificity and sensitivity of the method. Is accuracy enough? How does the patient benefit? The net benefit with DCA is defined as the proportion of true positives minus the proportion of false positives, weighted by the relative harm of falsepositive and false-negative results. As a consequence, DCA curves integrate the preferences of doctors or other decision-makers to select a high-risk threshold based on their preferences; for example, how worried they are about CVD underdiagnosis or overdiagnosis, allowing them to evaluate the benefit of the model at that threshold. ${ }^{61,62}$ Third, we visualized a predictive model with a nomogram and emphasized the integration of comprehensive patient information, which is often used in oncology to estimate prognosis. ${ }^{63}$ However, it is rare to apply nomograms in the cardiovascular and cerebrovascular fields. Nomograms are convenient when evaluating risk and provide superior information when determining the need for prevention and intervention in CVD. Therefore, nomograms can improve the understanding of CVD risk factors for both patients and doctors.

Although our methods offer powerful predictive performance, there are several limitations. First, our study population was from a high-risk area in northern China; therefore, caution is needed when generalizing our results to populations in other regions. To allow for more generalizable results, our team is expanding the scope of our research. Second, although we collected clinical information, such as stroke and coronary heart disease history, biochemical results, ECG results, and echocardiography results. We did not include rheumatoid arthritis, previous hypertensive pregnancy disorders, depression and abnormal $\mathrm{Q}$ wave in $\mathrm{ECG},{ }^{64}$ which may be important risk factors for CVD. Third, although a strict test was conducted after training investigators, we did not evaluate inter- and intra-rater variability for the investigators' scoring, we do not know the extent of measurement error.
Fourth, the follow-up period of 4.66 years was relatively short, which might have influenced the reliability of the results for the following reasons: it is hard to compare to a 10-year risk prediction setting which was most commonly used; we may only be capturing events early in a person's life, especially since our population was young, and those events might have different associations with CVD risk factors affecting our generalizability.

However, follow-up is on-going, and we will evaluate this prediction model in greater depth, in the future.

\section{Conclusion}

Using the updated incidence of CVD in the NCRCHS cohort, we established a new predictive model. This novel predictive model indicated that ECG and echocardiography indicators should be included in the prediction model to improve the assessment of CVD.

\section{Data Sharing Statement}

Data are available upon reasonable request.

\section{Ethics Approval}

This study was approved by the Ethics Committee of China Medical University (Shenyang, China), ID: 2018194. Written informed consent was obtained from all participants. Illiterate participants had their consents signed by close relatives.

\section{Acknowledgment}

We sincerely thank professor Jiang $\mathrm{He}$ of Tulane University for his guidance in research and design.

\section{Author Contributions}

All authors made a significant contribution to the work reported, including the conception, study design, execution, acquisition of data, analysis and interpretation, or in all these areas; have drafted or written, or substantially revised or critically reviewed the article; have agreed on the journal to which the article will be submitted; reviewed and agreed on all versions of the article; agree to take responsibility for the article.

\section{Funding}

This study was funded by the National Science and Technology Support Program of China (No. 2012BAJ18B08-7), the National Key Research and Development Program of China (No. 2017YFC1307600), the Shenyang Science and Technology Bureau (No.19- 
112-4-003), and the National Natural Science Foundation of China (No. 81970254).

\section{Disclosure}

The authors report no conflicts of interest in this work.

\section{References}

1. Doyle JT, Dawber TR, Kannel WB, et al. The relationship of cigarette smoking to coronary heart disease: the second report of the combined experience of the Albany, NY, and Framingham, Mass, Studies. JAMA. 1964;190(10):886-890. doi:10.1001/ jama.1964.03070230022006

2. Dawber TR, Kannel WB. The Framingham study. An epidemiological approach to coronary heart disease. Circulation. 1966;34 (4):553-555. doi:10.1161/01.cir.34.4.553

3. Kannel WB, Dawber TR, Kagan A, et al. Factors of risk in the development of coronary heart disease-six year follow-up experience. The Framingham Study. Ann Intern Med. 1961;55:33-50. doi:10.7326/0003-4819-55-1-33

4. Hilvo M, Meikle PJ, Pedersen ER, et al. Development and validation of a ceramide- and phospholipid-based cardiovascular risk estimation score for coronary artery disease patients. Eur Heart J. 2020;41 (3):371-380. doi:10.1093/eurheartj/ehz387

5. Araujo AB, Hall SA, Ganz P, et al. Does erectile dysfunction contribute to cardiovascular disease risk prediction beyond the Framingham risk score? J Am Coll Cardiol. 2010;55(4):350-356. doi:10.1016/j.jacc.2009.08.058

6. de Lemos JA, Ayers CR, Levine BD, et al. Multimodality strategy for cardiovascular risk assessment: performance in 2 population-based cohorts. Circulation. 2017;135(22):2119-2132. doi:10.1161/ circulationaha.117.027272

7. Eichler K, Puhan MA, Steurer J, et al. Prediction of first coronary events with the Framingham score: a systematic review. Am Heart $J$ 2007;153(5):722-31, 31.e1-8. doi:10.1016/j.ahj.2007.02.027

8. Conroy RM, Pyörälä K, Fitzgerald AP, et al. Estimation of ten-year risk of fatal cardiovascular disease in Europe: the SCORE project. Eur Heart $J .2003 ; 24(11): 987-1003$. doi:10.1016/s0195-668x(03)00114-3

9. Yang X, Li J, Hu D, et al. Predicting the 10-year risks of atherosclerotic cardiovascular disease in Chinese Population: the China-PAR Project (Prediction for ASCVD Risk in China). Circulation. 2016;134(19):1430-1440. doi:10.1161/ circulationaha.116.022367

10. Anand DV, Lim E, Hopkins D, et al. Risk stratification in uncomplicated type 2 diabetes: prospective evaluation of the combined use of coronary artery calcium imaging and selective myocardial perfusion scintigraphy. Eur Heart J. 2006;27(6):713-721. doi:10.1093/eurheartj/ehi808

11. Thom T, Haase N, Rosamond W, et al. Heart disease and stroke statistics-2006 update: a report from the American Heart Association Statistics Committee and Stroke Statistics Subcommittee. Circulation. 2006;113(6):e85-151. doi:10.1161/ circulationaha.105.171600

12. Huang Y, Luo J, Liu X, et al. Gamma-glutamyltransferase and risk of acute coronary syndrome in young Chinese patients: a Case-Control Study. Dis Markers. 2018;2018:2429160. doi:10.1155/2018/2429160

13. Nielsen JB, Graff C, Rasmussen PV, et al. Risk prediction of cardiovascular death based on the QTc interval: evaluating age and gender differences in a large primary care population. Eur Heart J. 2014;35 (20):1335-1344. doi:10.1093/eurheartj/ehu081

14. Tverdal A, Hjellvik V, Selmer R. Heart rate and mortality from cardiovascular causes: a 12 year follow-up study of 379,843 men and women aged 40-45 years. Eur Heart J. 2008;29(22):2772-2781. doi:10.1093/eurheartj/ehn435
15. Shah AJ, Vaccarino V, Janssens AC, et al. An electrocardiogram-based risk equation for incident cardiovascular disease from the national health and nutrition examination survey. JAMA Cardiol. 2016;1(7):779-786. doi:10.1001/jamacardio.2016.2173

16. Leigh JA, O’Neal WT, Soliman EZ. Electrocardiographic left ventricular hypertrophy as a predictor of cardiovascular disease independent of left ventricular anatomy in subjects aged $\geq 65$ years. Am J Cardiol. 2016;117(11):1831-1835. doi:10.1016/j.amjcard.2016.03.020

17. Gupta S, Berry JD, Ayers CR, et al. Left ventricular hypertrophy, aortic wall thickness, and lifetime predicted risk of cardiovascular disease: theDallas Heart Study. JACC Cardiovasc Imaging. 2010;3 (6):605-613. doi:10.1016/j.jcmg.2010.03.005

18. Li Z, Guo X, Liu Y, et al. The relation of moderate alcohol consumption to hyperuricemia in a rural general population. Int $J$ Environ Res Public Health. 2016;13(7). doi:10.3390/ijerph13070732

19. The National Essential Public Health Service Program Office for Management of Hypertension in Primary Health Care. Guidelines for primary hypertension prevention and management 2020 edition. Chinese Journal of Medical Frontiers.13.04(2021):26-37.doi:CNKI: SUN: yxqY.0.2021-04-007.

20. Hsu CY. CKD-EPI eGFR categories were better than MDRD categories for predicting mortality in a range of populations. Ann Intern Med. 2012;157(10):Jc5-12. doi:10.7326/0003-4819-157-10-20121120002012

21. Li Z, Liu Y, Guo X, et al. Long noncoding RNA myocardial infarction-associated transcript is associated with the microRNA-150-5p/ P300 pathway in cardiac hypertrophy. Int $J$ Mol Med. 2018;42 (3):1265-1272. doi:10.3892/ijmm.2018.3700

22. Postema PG, Wilde AA. The measurement of the QT interval. Curr Cardiol Rev. 2014;10(3):287-294. doi:10.2174/1573403×1066614051 4103612

23. Brink PA, Crotti L, Corfield V, et al. Phenotypic variability and unusual clinical severity of congenital long-QT syndrome in a founder population. Circulation. 2005;112(17):2602-2610. doi:10.1161/circulationaha.105.572453

24. Schouten EG, Dekker JM, Meppelink P, et al. QT interval prolongation predicts cardiovascular mortality in an apparently healthy population. Circulation. 1991;84(4):1516-1523. doi:10.1161/01. cir.84.4.1516

25. Goldenberg I, Horr S, Moss AJ, et al. Risk for life-threatening cardiac events in patients with genotype-confirmed long-QT syndrome and normal-range corrected QT intervals. J Am Coll Cardiol. 2011;57 (1):51-59. doi:10.1016/j.jacc.2010.07.038

26. Pelliccia A, Maron BJ, Culasso F, et al. Athlete's heart in women: echocardiographic characterization of highly trained elite female athletes. JAMA. 1996;276(3):211-215. doi:10.1001/jama.276.3.211

27. Konovalov DA, Bajema N, Litow B. Modified SIMPSON O(n3) algorithm for the full sibship reconstruction problem. Bioinformatics (Oxford, England). 2005;21(20):3912-3917. doi:10.1093/bioinformatics/bti642

28. Asplund K, Tuomilehto J, Stegmayr B, et al. Diagnostic criteria and quality control of the registration of stroke events in the MONICA project. Acta Med Scand Suppl. 1988;728:26-39. doi:10.1111/j.09546820.1988.tb05550.x

29. Smith SC Jr., Benjamin EJ, Bonow RO, et al. AHA/ACCF secondary prevention and risk reduction therapy for patients with coronary and other atherosclerotic vascular disease: 2011 update: a guideline from the American Heart Association and American College of Cardiology Foundation. Circulation. 2011;124(22):2458-2473. doi:10.1161/ CIR.0b013e318235eb4d

30. Solini A, Penno G, Bonora E, et al. Diverging association of reduced glomerular filtration rate and albuminuria with coronary and noncoronary events in patients with type 2 diabetes: the renal insufficiency and cardiovascular events (RIACE) Italian multicenter study. Diabetes Care. 2012;35(1):143-149. doi:10.2337/dc11-1380 
31. D’Agostino RB Sr., Vasan RS, Pencina MJ, et al. General cardiovascular risk profile for use in primary care: the Framingham Heart Study. Circulation. 2008;117(6):743-753. doi:10.1161/ circulationaha.107.699579

32. Alba AC, Agoritsas T, Walsh M, et al. Discrimination and calibration of clinical prediction models: users' guides to the medical literature. JAMA. 2017;318(14):1377-1384. doi:10.1001/jama.2017.12126

33. Pencina MJ, D’Agostino RB Sr., D’Agostino RB Jr., et al. Evaluating the added predictive ability of a new marker: from area under the ROC curve to reclassification and beyond. Stat Med. 2008;27(2):15772; discussion 207-12. doi:10.1002/sim.2929

34. Ma Q, Li Z, Guo X, et al. Prevalence and risk factors of prolonged corrected QT interval in general Chinese population. $B M C$ Cardiovasc Disord. 2019;19(1):276. doi:10.1186/s12872-019-1244-7

35. Li Z, Bai Y, Guo X, et al. Alcohol consumption and cardiovascular diseases in rural China. Int $J$ Cardiol. 2016;215:257-262. doi:10.1016/j.ijcard.2016.04.095

36. Li Z, Guo X, Liu Y, et al. Metabolism rather than obesity is associated with ischemic stroke: a cross-sectional study in rural Northeastern China. SpringerPlus. 2016;5(1):1419. doi:10.1186/ s40064-016-3088-2

37. Li Z, Guo X, Jiang H, et al. Diagnosed but not undiagnosed diabetes is associated with depression in rural areas. Int J Environ Res Public Health. 2016;13(11):1136. doi:10.3390/ijerph13111136

38. Muntner P, Colantonio LD, Cushman M, et al. Validation of the atherosclerotic cardiovascular disease Pooled Cohort risk equations. JAMA. 2014;311(14):1406-1415. doi:10.1001/jama.2014.2630

39. Gao XQ, Zhang YH, Liu F, et al. The piRNA CHAPIR regulates cardiac hypertrophy by controlling METTL3-dependent N(6)-methyladenosine methylation of Parp10 mRNA. Nat Cell Biol. 2020;22 (11):1319-1331. doi:10.1038/s41556-020-0576-y

40. Dekker JM, Crow RS, Hannan PJ, et al. Heart rate-corrected QT interval prolongation predicts risk of coronary heart disease in black and white middle-aged men and women: the ARIC study. $\mathrm{J}$ Am Coll Cardiol. 2004;43(4):565-571. doi:10.1016/j.jacc.2003.09.040

41. Kim NH, Pavkov ME, Nelson RG, et al. The separate and joint effects of prolonged QT interval and heart rate on mortality. Atherosclerosis. 2010;209(2):539-544. doi:10.1016/j. atherosclerosis.2009.09.026

42. Maebuchi D, Arima H, Doi Y, et al. QT interval prolongation and the risks of stroke and coronary heart disease in a general Japanese population: the Hisayama study. Hypertens Res. 2010;33 (9):916-921. doi:10.1038/hr.2010.88

43. Oka H, Mochio S, Sato K, et al. Prolongation of QTc interval and autonomic nervous dysfunction in diabetic patients. Diabetes Res Clin Pract. 1996;31(1-3):63-70. doi:10.1016/0168-8227(96)01194-1

44. Roden DM, Spooner PM. Inherited long QT syndromes: a paradigm for understanding arrhythmogenesis. J Cardiovasc Electrophysiol. 1999;10(12):1664-1683. doi:10.1111/j.1540-8167.1999.tb00231.x

45. Peters RW, Byington RP, Barker A, et al. Prognostic value of prolonged ventricular repolarization following myocardial infarction: the BHAT experience. The BHAT Study Group. J Clin Epidemiol. 1990;43(2):167-172. doi:10.1016/0895-4356(90)90180-w

46. Marfella R, Nappo F, De Angelis L, et al. The effect of acute hyperglycaemia on QTc duration in healthy man. Diabetologia. 2000;43(5):571-575. doi:10.1007/s001250051345

47. Dekker JM, Feskens EJ, Schouten EG, et al. QTc duration is associated with levels of insulin and glucose intolerance. The Zutphen Elderly Study. Diabetes. 1996;45(3):376-380. doi:10.2337/ diab.45.3.376
48. Festa A, D’Agostino R Jr., Rautaharju P, et al. Is QT interval a marker of subclinical atherosclerosis in nondiabetic subjects? The Insulin Resistance Atherosclerosis Study (IRAS). Stroke. 1999;30 (8):1566-1571. doi:10.1161/01.str.30.8.1566

49. Gastaldelli A, Emdin M, Conforti F, et al. Insulin prolongs the QTc interval in humans. Am J Physiol Regul Integr Comp Physiol. 2000;279(6):R2022-5. doi:10.1152/ajpregu.2000.279.6.R2022

50. Carella MJ, Mantz SL, Rovner DR, et al. Obesity, adiposity, and lengthening of the QT interval: improvement after weight loss. Int J Obes Relat Metab Disord. 1996;20(10):938-942.

51. Rautaharju PM, Park LP, Chaitman BR, et al. The Novacode criteria for classification of ECG abnormalities and their clinically significant progression and regression. J Electrocardiol. 1998;31(3):157-187. doi:10.1016/S0022-0736(98)90132-7

52. Capotosto L, Massoni F, De Sio S, et al. Early diagnosis of cardiovascular diseases in workers: role of standard and advanced echocardiography. Biomed Res Int. 2018;2018:7354691. doi:10.1155/2018/7354691

53. Utsunomiya H, Yamamoto $H$, Urabe $Y$, et al. Association between heart calcification assessed by echocardiography and future cardiovascular disease mortality and morbidity. Int J Cardiol Heart Vessel. 2013;2:15-20. doi:10.1016/j.ijchv.2013.11.007

54. Liu L, Yun C, Kuo J, et al. Aortic root remodeling as an indicator for diastolic dysfunction and normative ranges in asians: comparison and validation with multidetector computed tomography. Diagnostics. 2020;10(9):712. doi:10.3390/diagnostics 10090712

55. Hoang K, Zhao Y, Gardin JM, et al. LV mass as a predictor of CVD events in older adults with and without metabolic syndrome and diabetes. JACC Cardiovasc Imaging. 2015;8(9):1007-1015. doi:10.1016/j.jcmg.2015.04.019

56. Lin BY, Li P, Wu XD, et al. The relationship between homocysteine, blood pressure variability, and left ventricular hypertrophy in patients with essential hypertension: an observational study. Adv Ther. 2020;37(1):381-389. doi:10.1007/s12325-019-01154-7

57. Park SK, Jung JY, Kang JG, et al. Left ventricular geometry and risk of incident hypertension. Heart. 2019;105(18):1402-1407. doi:10.1136/heartjnl-2018-314657

58. Loncaric F, Nunno L, Mimbrero M, et al. Basal ventricular septal hypertrophy in systemic hypertension. Am J Cardiol. 2020;125 (9):1339-1346. doi:10.1016/j.amjcard.2020.01.045

59. Shadman R, Allison MA, Criqui MH. Glomerular filtration rate and $\mathrm{N}$-terminal pro-brain natriuretic peptide as predictors of cardiovascular mortality in vascular patients. J Am Coll Cardiol. 2007;49 (22):2172-2181. doi:10.1016/j.jacc.2007.02.047

60. Vickers AJ, Elkin EB. Decision curve analysis: a novel method for evaluating prediction models. Med Decis Making. 2006;26 (6):565-574. doi:10.1177/0272989x06295361

61. Fitzgerald M, Saville BR, Lewis RJ. Decision curve analysis. JAMA. 2015;313(4):409-410. doi:10.1001/jama.2015.37

62. Vickers AJ, van Calster B, Steyerberg EW. A simple, step-by-step guide to interpreting decision curve analysis. Diagn Progn Res. 2019;3:18. doi:10.1186/s41512-019-0064-7

63. Balachandran VP, Gonen M, Smith JJ, et al. Nomograms in oncology: more than meets the eye. Lancet Oncol. 2015;16(4):e173-80. doi:10.1016/s1470-2045(14)71116-7

64. Yang Y, Li W, Zhu H, et al. Prognosis of unrecognised myocardial infarction determined by electrocardiography or cardiac magnetic resonance imaging: systematic review and meta-analysis. BMJ (Clinical Research Ed). 2020;369:m1184. doi:10.1136/bmj.m1184 


\section{Publish your work in this journal}

Risk Management and Healthcare Policy is an international, peerreviewed, open access journal focusing on all aspects of public health, policy, and preventative measures to promote good health and improve morbidity and mortality in the population. The journal welcomes submitted papers covering original research, basic science, clinical \& epidemiological studies, reviews and evaluations, guidelines, expert opinion and commentary, case reports and extended reports. The manuscript management system is completely online and includes a very quick and fair peer-review system, which is all easy to use. Visit http://www.dovepress.com/testimonials.php to read real quotes from published authors.

Submit your manuscript here: https://www.dovepress.com/risk-management-and-healthcare-policy-journal 